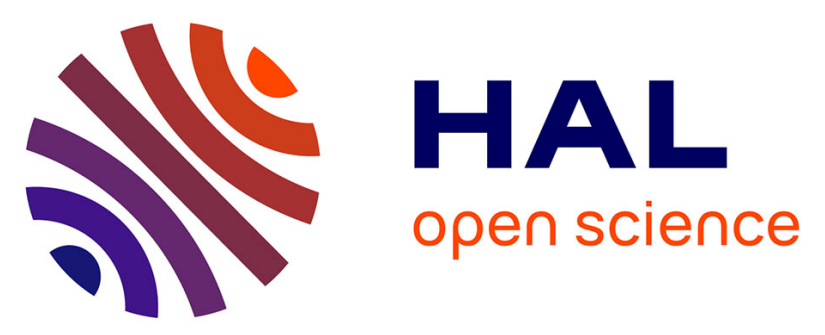

\title{
Col-OSSOS: Probing Ice Line/Colour Transitions within the Kuiper Belt's Progenitor Populations
}

Laura Buchanan, Megan Schwamb, Wesley Fraser, Michele Bannister, Michäel Marsset, Rosemary Pike, David Nesvorny, Johnj Kavelaars, Susan Benecchi, Matthew Lehner, et al.

\section{To cite this version:}

Laura Buchanan, Megan Schwamb, Wesley Fraser, Michele Bannister, Michäel Marsset, et al.. ColOSSOS: Probing Ice Line/Colour Transitions within the Kuiper Belt's Progenitor Populations. Europlanet Science Congress 2020, Sep 2020, virtuel, Germany. pp.EPSC2020-185, 10.5194/epsc2020-185 . hal-03572420

\section{HAL Id: hal-03572420 \\ https://hal.science/hal-03572420}

Submitted on 14 Feb 2022

HAL is a multi-disciplinary open access archive for the deposit and dissemination of scientific research documents, whether they are published or not. The documents may come from teaching and research institutions in France or abroad, or from public or private research centers.
L'archive ouverte pluridisciplinaire HAL, est destinée au dépôt et à la diffusion de documents scientifiques de niveau recherche, publiés ou non, émanant des établissements d'enseignement et de recherche français ou étrangers, des laboratoires publics ou privés.

\section{(ㄷ)(i)}

Distributed under a Creative Commons Attribution| 4.0 International License 
EPSC Abstracts

Vol. 14, EPSC2020-185, 2020

https://doi.org/10.5194/epsc2020-185

Europlanet Science Congress 2020

(C) Author(s) 2022. This work is distributed under

the Creative Commons Attribution 4.0 License.

\section{Col-OSSOS: Probing Ice Line/Colour Transitions within the Kuiper Belt's Progenitor Populations}

Laura Buchanan ${ }^{1}$, Megan Schwamb ${ }^{1}$, Wesley Fraser $^{2}$, Michele Bannister ${ }^{3}$, Michäel Marsset ${ }^{4}$, Rosemary Pike ${ }^{5}$, David Nesvorny ${ }^{6}$, Johnj Kavelaars ${ }^{2,7}$, Susan Benecchi ${ }^{8}$, Matthew Lehner ${ }^{9}$, Shiang-Yu Wang $^{9}$, Audrey Thirouin ${ }^{10}$, Nuno Peixinho ${ }^{11}$, Kathryn Volk ${ }^{12}$, Mike Alexandersen ${ }^{9}$, Ying-Tung Chen ${ }^{9}$, Audrey Delsanti ${ }^{13}$, Brett Gladman ${ }^{14}$, Stephen Gwyn ${ }^{2}$, and Jean-Marc Petit ${ }^{15}$

${ }^{1}$ Queen's University Belfast, Astrophysics Research Centre, United Kingdom of Great Britain and Northern Ireland (Ibuchanan14@qub.ac.uk)

${ }^{2}$ NRC-Herzberg Astronomy and Astrophysics, National Research Council of Canada, 5071 West Saanich Road, Victoria, BC V9E 2E7, Canada

${ }^{3}$ School of Physical and Chemical Sciences-Te Kura Matū, University of Canterbury, Private Bag 4800, Christchurch 8140, New Zealand

${ }^{4}$ Department of Earth, Atmospheric and Planetary Sciences, MIT, 77 Massachusetts Avenue, Cambridge, MA 02139, USA

${ }^{5}$ Harvard \& Smithsonian Center for Astrophysics, 60 Garden Street, Cambridge, MA 02138, USA

${ }^{6}$ Department of Space Studies, Southwest Research Institute, 1050 Walnut St., Suite 300, Boulder, CO, 80302, United States

${ }^{7}$ Department of Physics and Astronomy, University of Victoria, Elliott Building, 3800 Finnerty Road, Victoria, BC V8P 5C2, Canada

${ }^{8}$ Planetary Science Institute, 1700 East Fort Lowell, Suite 106, Tucson, AZ 85719, USA

${ }^{9}$ Institute of Astronomy and Astrophysics, Academia Sinica; 11F of AS/NTU Astronomy-Mathematics Building, No.1, Sec. 4, Roosevelt Rd Taipei 10617, Taiwan, R.O.C

${ }^{10}$ Lowell Observatory, 1400 W. Mars Hill Rd., Flagstaff, AZ 86001, USA

${ }^{11}$ CITEUC - Centre for Earth and Space Science Research of the University of Coimbra, Geophysical and Astronomical Observatory of the University of Coimbra, 3030-004 Coimbra, Portugal

${ }^{12}$ Lunar and Planetary Laboratory, University of Arizona, 1629 E. University Blvd., Tucson, AZ 85721, USA

${ }^{13}$ Aix Marseille Université, CNRS, LAM (Laboratoire d'Astrophysique de Marseille) UMR 7326, F-13388, Marseille, France

${ }^{14}$ Department of Physics and Astronomy, University of British Columbia, Vancouver, BC, Canada

${ }^{15}$ Institut UTINAM UMR6213, CNRS, Univ. Bourgogne Franche-Comté, OSU Theta F-25000 Besanc $\square$ on, France

The Colours of the Outer Solar System Origins Survey (Col-OSSOS, Schwamb et al., 2019) has examined the surface compositions of Kuiper Belt Objects (KBOs) by way of broadband g-, r- and Jband photometry, using the Gemini North Hawaii Telescope. This survey showed a bimodal distribution in the colours of the objects surveyed, consistent with previous colour surveys (Tegler et al., 2016). These broadband surface colours can be considered a proxy for surface composition of these KBOs, so this survey allows the frequency of different surface compositions within the outer Solar System to be explored. The bimodality of the observed colours suggests the presence of some sort of surface transition within the Kuiper belt, perhaps due to a volatile ice-line transition in the pristine planetesimal disk that existed before Neptune's migration. The Outer Solar System Origins Survey (OSSOS, Bannister et al., 2018), from which Col-OSSOS selected objects brighter than 23.6 r-band magnitude, has well characterised and quantified biases, so allowing for comparisons between the observations and numerical models of the Kuiper belt.

By applying different colour transitions to the primordial planetesimal disk, in this work we explore 
the possible positions for ice line/colour transitions within the planetesimal disk that existed before Neptune's migration. Within Schwamb et al. (2019), a simplified toy model was used to investigate the possible position of this transition. Nesvorny et al. (2020) has investigated the primordial colour fraction, in particular how it can create the inclination distribution that we see in the colours of KBOs today. In this work we use a full dynamical model of the Kuiper belt to more precisely pinpoint the possible location of this transition. We make use of the model by Nesvorny \& Vokrouhlicky (2016) of Neptune's migration from 23 au to $30 \mathrm{au}$, and the consequent perturbation of the Kuiper belt into its current form. This model allows precise tracking of the objects from their pre-Neptune migration to post-Neptune migration positions, allowing various colour transition positions in the initial disk, an example of which is shown in Figure 1, to be compared with the Col-OSSOS observations of the modern day disk.
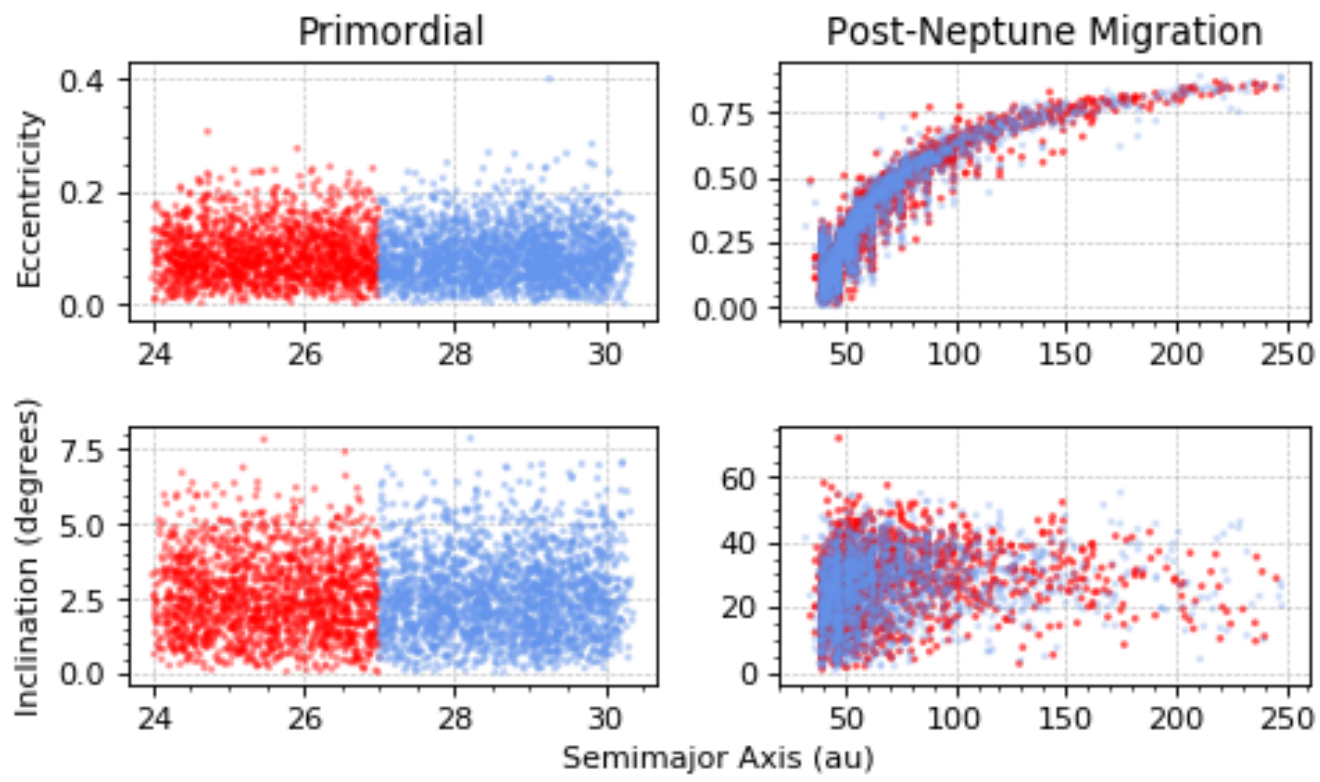

Figure 1: An example red/neutral transition at 27 au. The left plots show the objects in the primordial disk, while the right plots show the objects post-Neptune migration from the model of Nesvorny \& Vokrouhlicky (2016).

The OSSOS survey simulator (Lawler et al., 2018) can then be used to calculate which of the simulated objects could have been observed by OSSOS, and so selected by Col-OSSOS for surface colour observations. The colour transition within the initial disk, shown in Figure 1, is moved radially outwards through the disk and the corresponding outputs are compared with the Col-OSSOS colour observations to see which initial disk colour transition positions are consistent with the modern day Kuiper belt. We will present results combing an accurate dynamical model of the Kuiper Belt's evolution by Nesvorny \& Vokrouhlicky (2016) with Col-OSSOS photometry. We will explore multiple radial colour distributions in the primordial planetesimal disk and implications for the the positions of ice line/colour transitions within the Kuiper Belt's progenitor populations.

\section{References}

Bannister, M. T., Gladman, B. J., Kavelaars, J. J., et al. 2018, ApJS, 236, 18

Lawler, S. M., Kavelaars, J. J., Alexandersen, M., et al. 2018, Front. Astron. Space Sci., 5, 14

Nesvorny, D., Vokrouhlicky, D., Alexandersen, M., et al. 2020, AJ, in press

Nesvorny, D., \& Vokrouhlicky, D. 2016, ApJ, 825

Schwamb, M. E., Bannister, M. T., Marsset, M., et al. 2019, ApJS, 243, 12

Tegler, S. C., Romanishin, W., Consolmagno, G. J., \& J., S. 2016, AJ, 152, 210 
